\title{
Cutting through the Fat: Relationship Between Comorbidities and Arrhythmias in Hypertrophic Cardiomyopathy
}

\author{
Alan Sugrue ${ }^{1}$ and David Lin ${ }^{1}$ \\ ${ }^{1}$ Hospital of the University of Pennsylvania
}

November 15, 2021

\section{Cutting through the Fat: Relationship Between Comorbidities and Arrhythmias in Hyper- trophic Cardiomyopathy}

Alan Sugrue MBBCh, MSc; David Lin MD, FHRS

Cardiovascular Division, Perelman School of Medicine at the University of Pennsylvania, Philadelphia, PA

Manuscript word count: 1223

Conflicts of interest: none

Funding: none

Address for Correspondence:

David Lin, MD

Hospital of the University of Pennsylvania

3400 Spruce Street

9 Founders Pavilion

Philadelphia, PA 19104

Email: david.lin@pennmedicine.upenn.edu

Hypertrophic cardiomyopathy (HCM) is a commonly encountered inherited heart disease with an estimated prevalence of 1:200 to 1:500 ${ }^{1}$. Approximately 30-40\% of those patients diagnosed with HCM will experience adverse events (sudden cardiac death [SCD], heart failure, atrial fibrillation). Risk stratification for arrhythmias in HCM has been typically based upon five traditional noninvasive pillars (syncope, left ventricular hypertrophy $>30 \mathrm{~mm}$, non-sustained VT, family history of sudden cardiac death, and abnormal blood pressure response to exercise). There has been some evolution in our understanding of risk, with the demotion of some to risk modifiers and identification of new risk markers/predictive scoring strategies (LGE on CMR, apical aneurysms, EF <50\%). In HCM patients, comorbidities (obesity, hypertension, obstructive sleep apnea) have been identified as significant modifiers of disease penetrance, severity, and clinical course, particularly in relation to symptoms and heart failure ${ }^{2-4}$. The relationship of comorbidities to arrhythmias is less explored, but population-level data in non-HCM patients suggests that comorbidities can influence arrhythmia risk ${ }^{5,6}$. Therefore, in HCM patients, if there were a positive association, this would provide an additional tool to refine arrhythmia risk.

In this issue of the Journal of Cardiovascular Electrophysiology, Sridharan et al. ${ }^{7}$ explore and attempt to answer this important question - what is the association of comorbidities in HCM patients with arrhythmia risk? This paper studies a large cohort of 2269 patients of $>18$ years evaluated between 2004 and 2018. 
The mean age was $54 \pm 15$ years, with $1392(61 \%)$ male, and a follow-up average of $4.0 \pm 3.4$ years. 1702 (75\%) had [?]1 medical comorbidity, and $50 \%$ of patients had [?]2 comorbidities, most commonly obesity (43\%), hyperlipidemia (39\%), and hypertension (27\%). 198 (11\%) HCM patients developed new-onset AF over follow-up at a rate of $2.6 \%$ per year. On univariate analysis, obesity was associated with a 1.7 -fold increase in risk for $\mathrm{AF}$ development compared to patients with normal BMI (95\% CI 1.0, 2.7, $\mathrm{p}=0.03$ ). In addition, $12 \%$ of obese patients developed AF (rate of $3.3 \%$ year) compared to just $7 \%$ with normal BMI (rate of $1.6 \% /$ year; $\mathrm{p}=0.006$ ). No other comorbidities were significant by univariate analysis. However, importantly on multivariate analysis, obesity did not show an association with AF (OR 1.3 [95\% CI 0.95, 1.79, $\mathrm{p}=0.10)$. Concerning SCD, $72(3 \%)$ of patients experienced a SCD event during the following up period $(0.8 \% /$ year $)$. There was no association between any comorbidities and the risk of SCD. While this is a large cohort study, the major limitation that one must consider is that past medical history was assessed at the initial clinical evaluation. It, therefore, does not reflect the development of new comorbidities over the follow-up period (which may have a different impact on arrhythmia risk). Additionally, a major comorbidity, obstructive sleep apnea, was not examined, which has important pathogenesis links to arrhythmias. Lastly, the number of patients with adjudicated SCD was low and may be underpowered to detect associations between comorbidities and ventricular arrhythmias.

The authors should be congratulated for completing this important study which demonstrates that in this cohort of HCM patients, comorbidities did not play a significant role in risk of arrhythmias (atrial or ventricular). This adds and confirms previous publications that failed to establish a strong association between obesity and adverse arrhythmic outcomes ${ }^{3,8}$. The data presented in this paper is important and surprising. The lack of association with arrhythmic risk given the plethora of data on patient comorbidities in non-HCM cohorts, and especially considering that obesity in HCM can lead to a more severe phenotype and worse disease progression ${ }^{9}$ is not entirely understood and requires further exploration.

One potential explanation is the application of body mass index (BMI) to define obesity. Although BMI is largely ingrained as the most conventional and convenient method to define obesity, it can be an imprecise measurement as it defines overall adiposity and doesn't consider the different elements of adiposity distribution such as abdominal vs. epicardial adipose tissue (EAT). EAT is an evolving area of interest given its proximity to cardiac tissue, and its presence and size have been associated with cardiac arrhythmias, particularly atrial fibrillation ${ }^{10}$. EAT is proposed to play a role in arrhythmogenesis by altering the electrical and structural properties of the cardiac myocardium ${ }^{11}$. Specifically, direct infiltration and promotion of fibrosis can set up the correct environment for reentry. Further, paracrine effects by adipokines can lead to ion channel modulation and alteration of cell-cell electrical coupling. Our knowledge of the role of EAT in HCM is limited. Muhib et al. ${ }^{12}$ in a cohort of 62 patients, showed an increased incidence of AF related to an increase in EAT, even when adjusted for baseline characteristics. Further examination of this in larger cohorts may be insightful.

Overall, the authors provide a thought-provoking paper that adds critical data to the HCM literature. As we broaden our understanding of the elements and measurement of obesity and other comorbidities, perhaps it will become an important risk stratification tool. Until then, the role of comorbidities and its impact on $\mathrm{AF}$ or SCD in this subset of patients with HCM remains unclear and further research is warranted.

\section{References:}

1. Ommen SR, Mital S, Burke MA, et al. 2020 AHA/ACC Guideline for the Diagnosis and Treatment of Patients With Hypertrophic Cardiomyopathy: A Report of the American College of Cardiology/American Heart Association Joint Committee on Clinical Practice Guidelines. J Am Coll Cardiol. 2020;76(25):e159e240.

2. Finocchiaro G, Magavern E, Sinagra G, et al. Impact of Demographic Features, Lifestyle, and Comorbidities on the Clinical Expression of Hypertrophic Cardiomyopathy. J Am Heart Assoc. 2017;6(12).

3. Olivotto I, Maron BJ, Tomberli B, et al. Obesity and its association to phenotype and clinical course in hypertrophic cardiomyopathy. J Am Coll Cardiol. 2013;62(5):449-457. 
4. Nerbass FB, Pedrosa RP, Danzi-Soares NJ, Drager LF, Arteaga-Fernandez E, Lorenzi-Filho G. Obstructive sleep apnea and hypertrophic cardiomyopathy: a common and potential harmful combination. Sleep Med Rev. 2013;17(3):201-206.

5. Javed S, Gupta D, Lip GYH. Obesity and atrial fibrillation: making inroads through fat. Eur Heart J Cardiovasc Pharmacother.2021;7(1):59-67.

6. Linz D, McEvoy RD, Cowie MR, et al. Associations of Obstructive Sleep Apnea With Atrial Fibrillation and Continuous Positive Airway Pressure Treatment: A Review. JAMA Cardiol. 2018;3(6):532-540.

7. Sridharan A. Impact of Comorbidities on Atrial Fibrillation and Sudden Cardiac Death in Hypertrophic cardiomyopathy. Journal of Cardiovascular Electrophysiology 2021.

8. Fumagalli C, Maurizi N, Day SM, et al. Association of Obesity With Adverse Long-term Outcomes in Hypertrophic Cardiomyopathy. JAMA Cardiol. 2020;5(1):65-72.

9. Nollet EE, Westenbrink BD, de Boer RA, Kuster DWD, van der Velden J. Unraveling the GenotypePhenotype Relationship in Hypertrophic Cardiomyopathy: Obesity-Related Cardiac Defects as a Major Disease Modifier. J Am Heart Assoc. 2020;9(22):e018641.

10. Vyas V, Hunter RJ, Longhi MP, Finlay MC. Inflammation and adiposity: new frontiers in atrial fibrillation. Europace.2020;22(11):1609-1618.

11. Ernault AC, Meijborg VMF, Coronel R. Modulation of Cardiac Arrhythmogenesis by Epicardial Adipose Tissue: JACC State-of-the-Art Review. J Am Coll Cardiol. 2021;78(17):1730-1745.

12. Muhib S, Fujino T, Sato N, Hasebe N. Epicardial adipose tissue is associated with prevalent atrial fibrillation in patients with hypertrophic cardiomyopathy. Int Heart J. 2013;54(5):297-303. 